\title{
DESIGN OF INTELLIGENT ALGORITHM BASED AIR VOLUME CONTROL SYSTEM FOR CENTRAL AIR CONDITIONING
}

\author{
Min Niu ${ }^{1}$, Quoc Tien Le'2, Rd Rohmat Saedudin ${ }^{3}$ \\ ${ }^{1}$ Shandong Vocational College of Industry, Zibo, 256414, Shandong, China \\ ${ }^{2}$ Haiphong Department of Education and Training, Haiphong, Vietnam \\ ${ }^{3}$ School of Industrial Engineering, Telkom University, 40257, Bandung, West Java, Indonesia \\ Email: niuniumin11@163.com
}

\begin{abstract}
This research aims to analyze the optimization value of intelligent control algorithm in the design of automatic variable air volume control system of central air conditioning, to improve the technology of central air conditioning and reduce the energy consumption of air conditioning. In this research, starting from analyzing the operation characteristics of variable air volume system of central air conditioning, a controller based on fuzzy intelligent control algorithm is proposed, and a terminal controller model based on intelligent control algorithm is established to design a new variable air volume automatic control system for central air conditioning. Besides, its performance is compared with that of central air conditioning system based on traditional proportion integration differentiation (PID) control algorithm. The results show that the automatic variable air volume control system of central air conditioning designed in this research based on the intelligent control algorithm has better energy saving effect. The application of fuzzy intelligent control algorithm improves the performance of the control system, reduces the power consumption of the central air-conditioning system, and optimizes the energy utilization efficiency. In this study, the intelligent control technology has been expanded to the national call for energy conservation and emission reduction, and the application of intelligent control algorithm to the variable air volume control system of central air conditioning has important practical significance, laying a foundation for the development of follow-up high-performance air conditioning system.
\end{abstract}

Keywords: Central Air Conditioning System, Intelligent Control Algorithm, Variable Air Volume Automatic Control, Energy Saving.

\section{Introduction}

With the rapid development of science and technology and the economy, people's living standards are improving day by day. Central air conditioning has become an essential infrastructure in large urban buildings, and its utilization rate is increasing significantly. In terms of building energy consumption, central air conditioning system occupies the most substantial proportion [1]. Global warming is becoming more and more dangerous for all living beings. Energy conservation and emission reduction have become the focus and hot issue in recent years. The critical way of building energy saving is to reduce the energy consumption of the central air conditioning system. Therefore, the question to reduce the energy consumption of a central air conditioning system is crucial to the energy conservation of buildings. The design of intelligent central air conditioning control system has been the research direction of many researchers. The intelligent control system has incomparable advantages in reducing building energy consumption and improving the energy utilization efficiency of the building [2,3].

Due to the gigantic central air conditioning system, low system efficiency, slow response, and complicated control are the main problems faced due to central air conditioning. At present, most of China's central air conditioning still adopts the traditional control technology, among which PID control technology is the most common. However, the adaptability of PID control technology is poor, and the control process is too rigid. Once the control parameters are set irrationally, the controlled inertia of the system will be too large, or the control force will be insufficient. Therefore, the temperature control effect of the central air conditioning system is volatile, and the unstable temperature control effect will significantly increase the energy consumption of the central air conditioning system [4]. To solve this problem, intelligent control technology came into being, which combines computer technology, information theory, electronic technology, control theory, and artificial intelligence algorithm. Compared with the traditional control 
algorithm, the intelligent control technology has a better processing effect in the complex and changeable environment, which effectively promotes the development of the control technology. A series of control algorithms are derived from intelligent control technology, such as fuzzy control algorithm, genetic evolution control algorithm, and artificial neural network control algorithm, etc. [5, 6].

The stable operation of central air-conditioning water system is always the main difficulty in the control industry. Li (2019) proposed the use of feedforward coupling compensation for water systems with multivariable, strongly coupled, nonlinear, and long delay characteristics to eliminate the coupling effect between temperature and pressure, and designed the Elman neural network controller for the first time.

Simulation results showed that the response time of the Elman neural network controller applied in the central air conditioning water system was shorter, the system was more stable, and the overshoot was small [7]. Sun (2017) et al. adopted the fuzzy PID control method instead of the traditional PID control method to meet the nonlinear requirements of the central air conditioning system, and constructed the variable frequency central air conditioning control system. This system can realize the independent regulation of variable water and variable air volume, thus achieving good energy saving effect [8]. To sum up, it can be concluded that at present, there are few studies on the application of intelligent control algorithm to variable air volume control system of central air conditioning.

To solve the problem of energy consumption of central air conditioning, in this research, it starts from the terminal control device in the variable air volume system of central air conditioning, analyzes the system characteristics of the variable air volume of central air conditioning, adopts the fuzzy intelligent control algorithm, and designs a new automatic control system of the variable air volume of central air conditioning.
The introduction of fuzzy intelligent control algorithm improves the performance of the system, improves the energy efficiency of the central air conditioning system, and achieves the goal of high efficiency and energy saving of the central air conditioning system. It is expected that the results of this study can provide technical support for the later intelligent building with energy saving and low consumption.

\section{Methodology}

\subsection{The principle and implementation process of fuzzy intelligent control algorithm}

The traditional variable air volume of central air conditioning is mainly based on the PID control algorithm to design the terminal controller of central air conditioning, but the PID control algorithm is not suitable for complex or changeable environments [9]. In recent years, intelligent fuzzy control algorithm has been gradually applied to the terminal controller of variable air volume central airconditioning system. The fuzzy intelligent control algorithm is a kind of control algorithm that fuses a fuzzy control system and neural network. It integrates the advantages of fuzzy control algorithm and artificial neural network. The main body of the fuzzy intelligent control algorithm is neural network, and it has the ability of fuzzy weight coefficient and fuzzy processing of sensor input.

In this research, assuming the fuzzy set $X, Y \in M(U), X \cup Y$ and $X \mathrm{I} Y$ are defined as the union and intersection of the fuzzy set $\mathrm{X}$ and $\mathrm{Y}$, and $\mathrm{X}^{\mathrm{c}}$ is defined as the complement of the fuzzy set $X$. The logical operation expression of its membership function is as follows:

$$
\begin{aligned}
& (X \mathrm{U} Y)(u)=X(u) \vee Y(u)=\max (X(u), Y(u)) \\
& (X \mathrm{I} Y)(u)=X(u) \wedge Y(u)=\min (X(u), Y(u))
\end{aligned}
$$

$$
X^{C}(u)=1-X(u)
$$

In this research, the fuzzy integral algorithm used by fuzzy intelligent control is as follows. $F: I \rightarrow E^{1}$

is set as a fuzzy numerical function on the interval $I$, and the fuzzy integral $\int_{1} F(t) d t$ of the function $F$ on the interval $I$ is defined as follows.

$$
\left[\int_{1} F(t) d t\right]^{\alpha}=\int_{1} F(t) d t=\left\{\int_{1} \mathrm{~g}(t) d t: g \in S\left(\mathrm{~F}_{\alpha}\right)\right\}, \alpha \in(0,1]
$$

Where, $S\left(F_{\alpha}\right)$ is the integrable selection of all $F_{\alpha}$ of the set-valued map.

$$
F_{\alpha}(t):=[F(t)]=\{x \in R \mid F(t)(x) \geq \alpha\}, t \in I
$$


The fuzzy intelligent control system can approximate the continuous nonlinear function on a subset, and the approximation error can be

$$
f(x)=\frac{\sum_{i=1}^{n} y^{l}\left[\prod_{i=1}^{n} a_{i}^{l} \exp \left(-\left(\frac{x_{i}-x_{i}^{l}}{\sigma_{i}^{l}}\right)^{2}\right)\right]}{\sum_{i=1}^{n}\left[\prod_{i=1}^{n} a_{i}^{l} \exp \left(-\left(\frac{x_{i}-x_{i}^{l}}{\sigma_{i}^{l}}\right)^{2}\right)\right]}
$$

Among them, $\quad l=1,2, \ldots \mathrm{M}, \quad a_{i}^{l} \in(0,1]$ $\sigma_{i}^{l} \in(0, \infty), x_{i}^{l}, y^{l} \in R$

The framework of fuzzy control system based on fuzzy intelligent control algorithm is shown in figure 1 . controlled in any close and small neighbourhood of the zero value, as shown in equation (6).
The fuzzy control system is composed of different types of sensors, generalized controlled objects, and fuzzy controllers. By adopting fuzzy rules, the fuzzy quantity can be changed into an exact quantity through fuzzification and defuzzification, to realize the precise control of the equipment

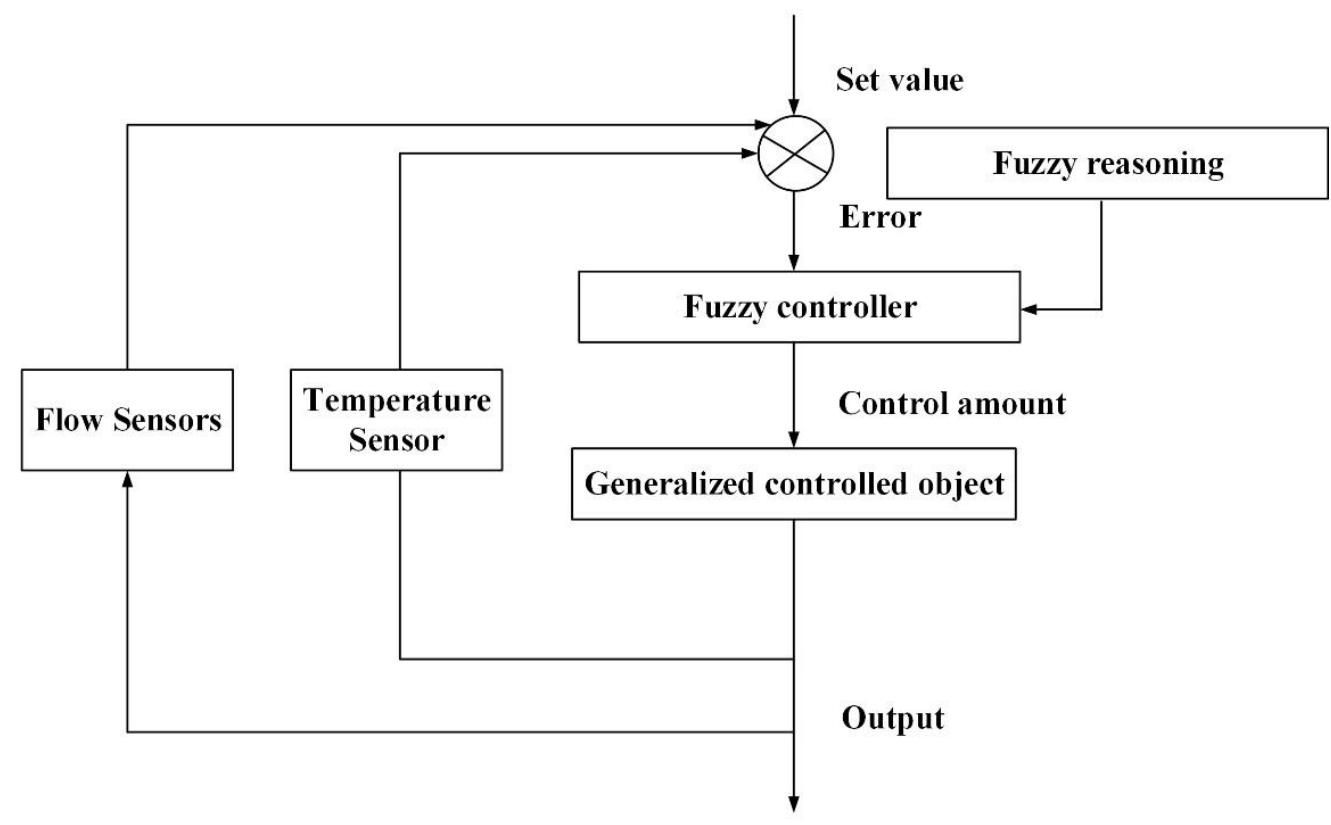

Figure 1: Framework diagram of fuzzy control system

\subsection{Design of variable air volume automatic control system for central air conditioning}

The central air-conditioning system is a device that satisfies people to make the temperature and humidity of the rooms in the building reach a particular requirement. It consists of various sensors, hot and cold media transmission and distribution, control systems, and other auxiliary equipment. Generally, the central air conditioning system is composed of the following parts, namely, the working area, the air handling part, the transmission and distribution of cold and hot media, the air conditioning control system, and the cold and hot source. The specific frame diagram of the central air conditioning system is shown in figure 2 . In summer, open cooling water circulation system and closed cooling water circulation system is used for cooling of central air conditioning system. The system uses water pumps to feed the cooling water provided by the main engine of the refrigerator into the fan coil tube of the floor. The purpose of water pumps is to work when the temperature drops, the aluminum sheet between the fan coil of the floor can effectively exchange heat with the high-temperature air circulating into the outdoor air, and then the cold air obtained can be sent into the indoor air for cooling. To ensure that the indoor temperature is kept within a very comfortable range in winter, when the indoor temperature is too low, the central air conditioner will send circulating hot water into the fan coil of the floor, and then circulate the lowtemperature outdoor air. In the case of low temperature air, the aluminum sheet of fan coil is also used to transfer heat to the low temperature air, which can promote the low temperature air to be sent into the room after heating, and finally effectively raise the indoor temperature. 


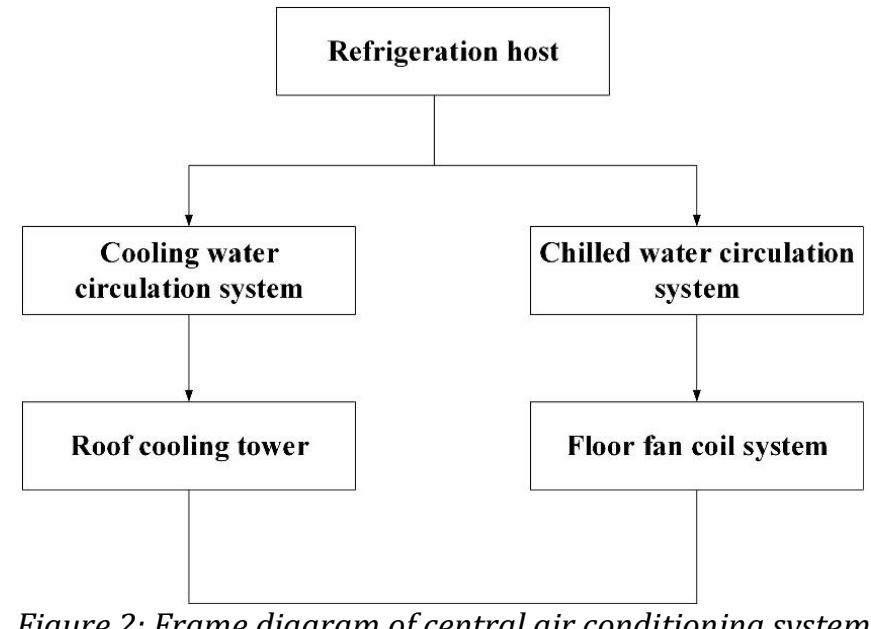

The automatic control system of the variable air volume of central air conditioning is composed of water system and air system. The function of the water system in the automatic control system of variable air volume of central air conditioning is the same as that in the central air conditioning system of fixed air volume. The operation principle of the variable air volume system of central air conditioning is to adjust the inlet air volume of the wind valve at the end. When the air parameters in the room change, the wind volume at the end will automatically improve the air volume according to the indoor conditions, to achieve the air volume load matching the room and control the indoor environment within the preset range.

The central air-conditioning variable air volume system must have sufficient treated air during operation to ensure that the temperature and humidity required for the room can be effectively adjusted. The relationship between air volume and indoor temperature and humidity is shown in equation (7). In the automatic control system of variable air volume of central air conditioning, the air supply volume in different air conditioning areas is changed by controlling the air valve. On the whole, speed regulation of frequency conversion of a fan is conducted to control the air volume of the total system. These two parameters cooperate to control the temperature and humidity of each area covered by the air conditioner.

$$
X=\frac{W_{q}}{\rho\left(I_{n}-I_{s}\right)}=\frac{W_{q}}{\rho c\left(T_{n}-T_{s}\right)}
$$

Where, $X$ is the air volume sent in per unit time, $W_{q}$ is the waste heat of total heat, $W_{s}$ is waste heat of sensible heat, $\rho$ is the density of the air in the room, $I_{n}$ is the air enthalpy in the room, $I_{s}$ is the enthalpy value of the air sent into the room by the air conditioning system, $\mathrm{c}$ is the specific heat capacity of air, $T_{n}$ is the temperature in the room, and $T_{s}$ is the temperature of the air sent into the room by the air conditioning system.

\subsection{Construction of fuzzy model of variable air volume control system for central air conditioning}

The terminal air volume control part of the variable air volume automatic control system for central air conditioning is a nonlinear large inertia multiple input and output systems. As the indoor environment is changeable, and the air temperature and humidity are complex, fuzzy intelligent controller is adopted to control it in this research. The main advantages of the control system include strong anti-interference ability, fast processing speed, and high air temperature and humidity. The structure of the fuzzy control system of variable air volume at the end of the central air conditioner is shown in figure 3. After the indoor air temperature is obtained by the temperature sensor placed in the room, it is compared with the set temperature to calculate the temperature difference. The data collected and calculated by the fuzzy intelligent controller are divided into discrete parts and then are blurred. The fuzzy intelligent controller makes a decision based on the fuzzy reasoning of the set rules, and outputs the exact value after the decision result is de-fuzzy.

The temperature difference collected by the fuzzy intelligent controller is set as E, and the adjusting quantity of the air valve is 0 , then the system operation process meets the following equation.

$$
\begin{aligned}
& E=T_{r}-T_{t} \\
& \Delta E=\frac{\left(E-E^{*}\right)}{t}
\end{aligned}
$$

Among them, $T_{r}$ is the actual indoor temperature collected by the sensor, $T_{t}$ is the set temperature of the room, $E^{*}$ is the temperature difference collected in the previous cycle, $\mathrm{t}$ is the time difference between the two temperature acquisitions. The fuzzy intelligent controller firstly fuzzy the $E$, then calculates and adjusts the air valve volume 0 according to the subset of rules of different grades, and finally corrects it, and sends the result to the air valve control execution system, thus completing the control of the air volume. 


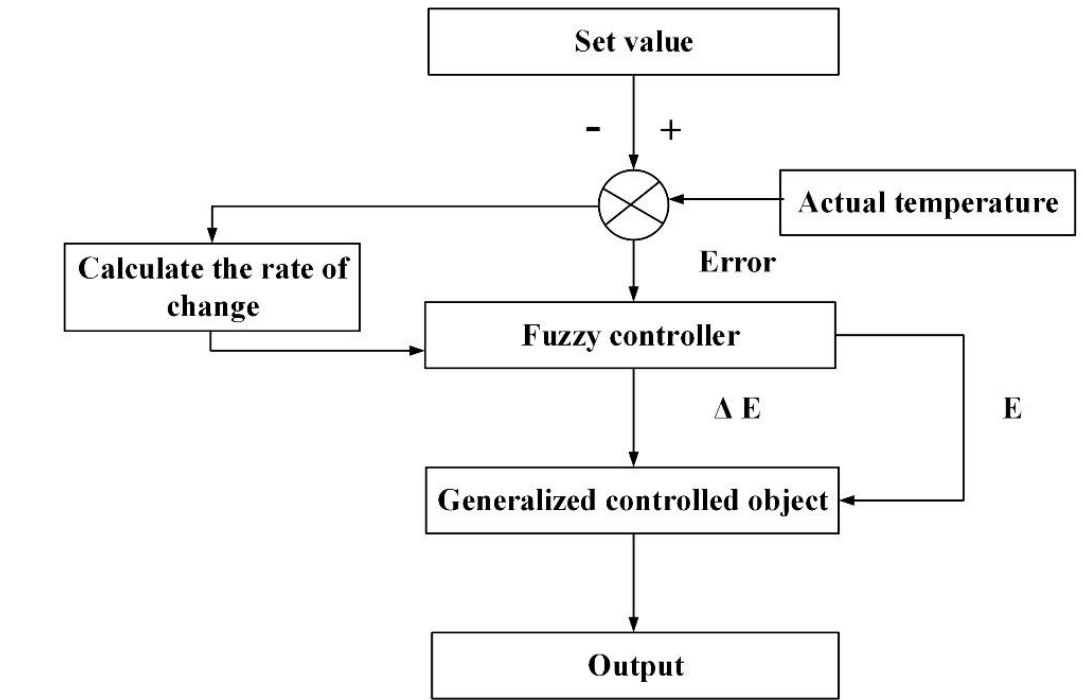

Figure 3: Structure diagram of terminal variable air volume fuzzy control system

\section{Results}

\subsection{The overall framework of the design of the variable air volume automatic control system for central air conditioner}

In this research, the overall framework of variable air volume automatic control system for central air conditioning based on intelligent control algorithm is shown in figure 4 . As can be observed from figure 4, the total system is composed of six parts: terminal display and control part, sensor, controller, control output driver, communication part, and upper computer. The terminal display and control part provides the current air parameter data and control input for each air conditioning area. The sensors include a temperature sensor and the angle sensor. The former is arranged in the whole area covered by the air conditioner to detect the air parameters in the air conditioner area.
The acquisition process of the temperature sensor is controlled by a microprogrammed control unit (MCU) in the terminal controller. The latter is installed on the air valve, the degree of opening and closing parameters of the air valve is measured and transmitted to the terminal controller. The terminal controller uses single-chip microcomputer as the control chip, which can control the air valve according to the temperature set by the operator based on the temperature of the air conditioning area collected by the sensor and combined with the fuzzy intelligent control algorithm. The control output drive is used to provide the external output drive, which isolates the terminal controller from the wind valve, thus improving the operation stability of the terminal controller. The communication part uses CAN bus as the communication mode. The software running in the upper computer completes the overall monitoring and control of the central air conditioning system.

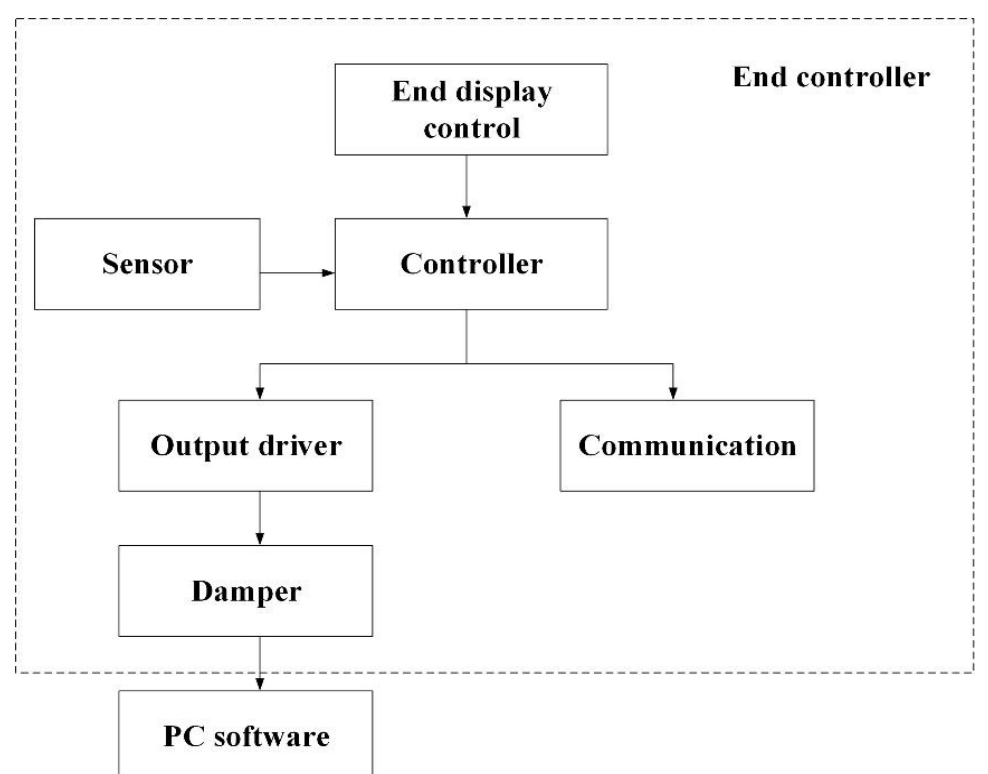

Figure 4: Structural diagram of the central air conditioning system with terminal variable air volume 


\subsection{Equipment selection of variable air volume automatic control system of central air conditioner}

In the automatic control system of variable air volume of central air conditioning based on intelligent control algorithm, the single clock/machine cycle (1T) single chip microcomputer produced by Hongjing technology company is adopted, and its model is STC15F2K60S2.
This microcontroller is a new generation 8051 microcontroller with high speed / low power consumption and super anti-interference.

The working voltage is $5 \mathrm{~V}$, it has eight-way highspeed 10-bit A/D conversion channel, it can directly connect and use the sensor of analog quantity, and the working frequency can reach up to $35 \mathrm{MHZ}$. The physical object and pin diagram of the MCU are shown in figure 5 .

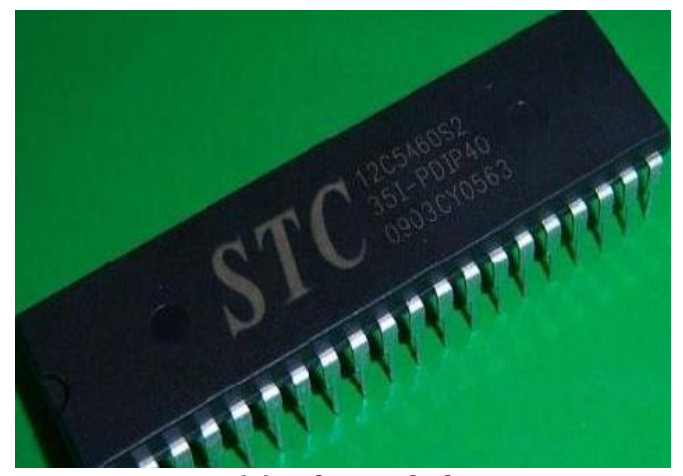

(a) Physical object

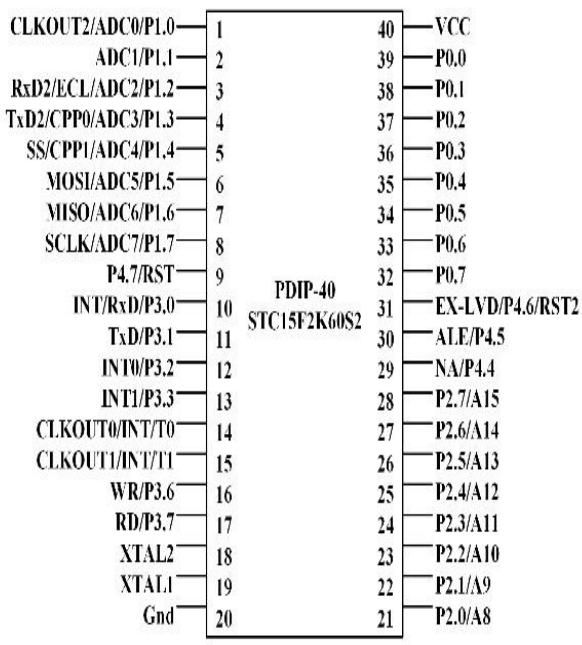

(b) Pin diagram

Figure 5: The physical and pin diagram of $M C U$

The temperature sensor uses platinum resistance PT100 produced by Shanghai Weilian. The resistance value of PT100 sensor will change with the change of temperature, and the temperature and resistance values are linearly correlated. PT100 has simple structure, good anti-interference ability, wide temperature range, low price and durability.
It does not need to use the external power supply. It can directly calculate the temperature value through A/D conversion channel of STC15F2K60S2 microcontroller. In this research, the application circuit of the temperature sensor in the design system is shown in figure 6.

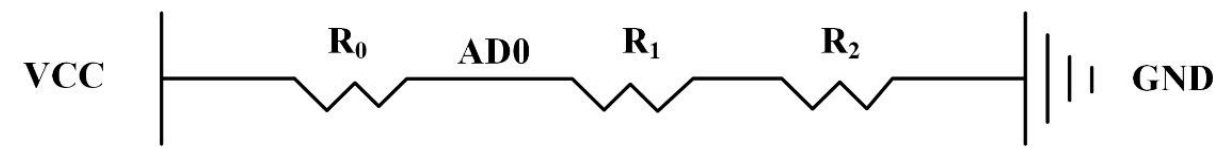

Figure 6: Application circuit diagram of temperature sensor

In this system, the magnetic sensitive angle sensor is adopted, which has strong environmental applicability, no contact and no friction, high reliability, long service life, and other characteristics, and it can be used in a variety of harsh environments, which is very suitable for this system. 
Its model is DWQT-V-180-2-G, which uses 5V DC analog voltage output, and the measurement angle range is $0-180^{\circ}$. The adopted CAN bus controller is produced by Semiconductors, its model is SJA1000, the controller supports the basic CAN bus mode and conforms to the CAN2.0 specification, and its pin diagram is shown in figure 7.

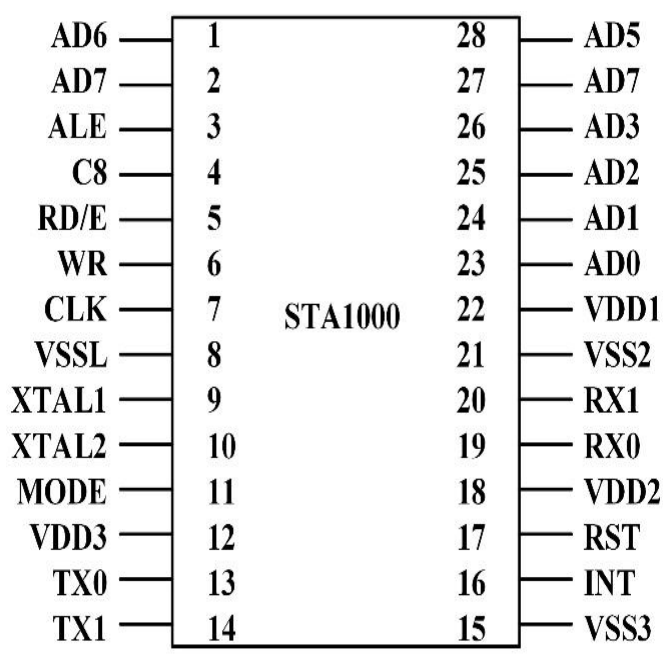

Figure 7: Pin diagram of CAN

\subsection{Test of variable air volume automatic control system of central air conditioner based on intelligent control algorithm}

The research is adopted to test the energy-saving performance of the automatic control system of variable air volume of central air conditioning based on the intelligent control algorithm. At the same time, the control system of central air conditioning is compared with the traditional PID controller, and two groups of experiments are designed. First, with the room of the same area as the main body, an air supply port is arranged to test the energy consumption of the two control systems in the daily use of air conditioning, to reflect the energy-saving effect of the variable air volume automatic control system of central air conditioning designed in this research. During the measurement, it is necessary to ensure that the running time of the air conditioning system is the same, and the opening and closing time are similar when coming in and out. By continually changing the number of coming in and out of personnel, the power consumption of the air conditioning system is measured.

The test results are shown in figure 8. Two rooms with the same experimental environment are taken, and the traditional control system and the system designed in this research are used to adjust the temperature of the two rooms to be same. After the temperature stabilizes, the temperature difference is continuously adjusted, and the power consumption during the adjustment is recorded. The statistical results are shown in figure 9.

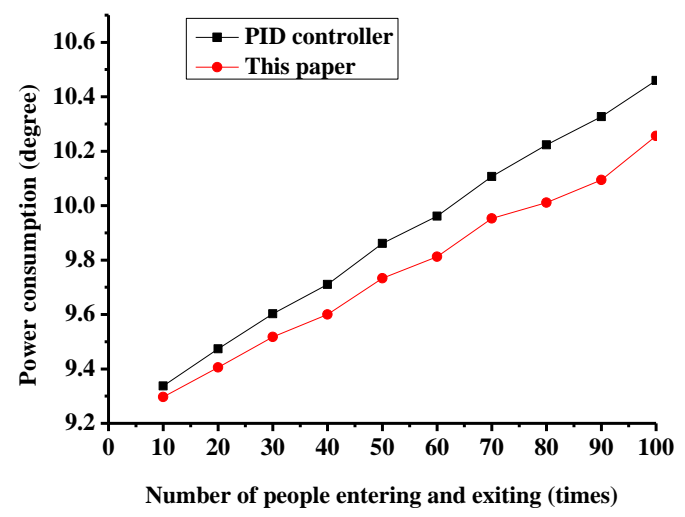

Figure 8: The relationship between the frequency of room access and the power consumption of air conditioning

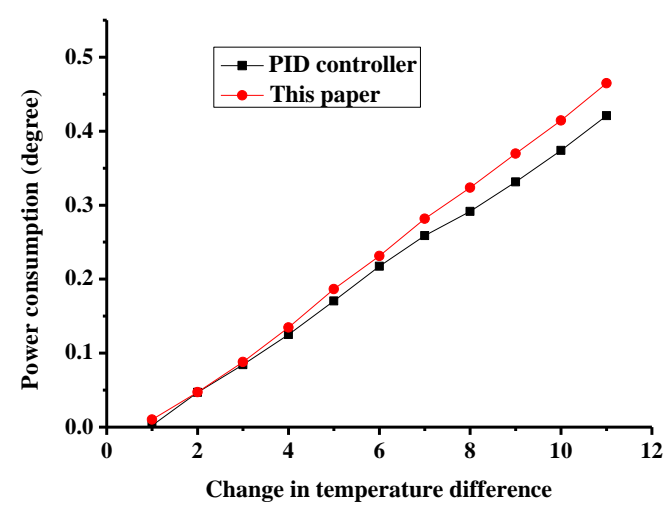

Figure 9: A comparison chart of power consumption of air conditioner when temperature changes

\section{Discussion}

The automatic control system of variable air volume of central air conditioning is divided into three parts: air supply system, return air matching system, and fresh air system. In the automatic control system of variable air volume of central air conditioning, the following regulation rules can be obtained according to the test results. If the actual temperature is much higher than the target temperature, and the indoor temperature is still rising fast, it is necessary to open the cold air valve and close the hot air valve to the maximum extent. If the actual temperature is much higher than the target temperature, and the indoor temperature is still rising slowly, it needs to close the hot air valve and open the cold air valve moderately. If the actual temperature is not much higher than the target temperature, and the indoor temperature change range is not extensive, then it should close the hot air valve and adjust the cold air valve to a small opening. If the actual temperature is a little higher than the target temperature, and the indoor temperature changes dramatically, then it should adjust the cold and hot air valve in the direction of restraining the change. 
If the actual temperature is lower than the target temperature, the above rules need to be applied in reverse.

By testing the energy-saving performance of the automatic control system of variable air volume of central air conditioning based on the intelligent control algorithm; it can be observed that in rooms having the same environment, there is no significant difference in the power consumption. between the PID controller and the fuzzy intelligent controller. Same was observed under static conditions and the power consumption of the two systems are roughly similar. However, after personnel come in and go out, the power consumption gap between the two will gradually become more extensive, and the power consumption is linearly related to the number of opening and closing times when personnel come in and go out. By continually adjusting the temperature difference in the room, the energy saving effect of fuzzy intelligent controller is still better than the traditional PID controller.

Meanwhile, the power consumption of fuzzy intelligent controller increases slowly, while the power consumption of PID controller increases fast. Therefore, the variable air volume automatic control system of central air conditioning based on intelligent control algorithm can better adapt to the complex and changeable indoor environment and has an excellent energy-saving effect. Compared with the control system of central air conditioning proposed by Kosaka et al. and Liu et al., the automatic control system of the variable air volume of central air conditioning based on the intelligent control algorithm in this research has better energy saving performance, better control effect, and can adapt to the complex and changeable environment [10-16].

\section{Conclusions}

The central air-conditioning system in the building occupies a large proportion in the building energy consumption system, and reducing the energy consumption of central air conditioning system can effectively reduce the energy consumption of the building. In this research, taking the terminal control device in the variable air volume system of central air conditioning as the starting point, the system characteristics of the variable air volume of central air conditioning are analyzed. The fuzzy model of variable air volume control system of central air conditioning is constructed by fuzzy intelligent control algorithm, and a new automatic control system of variable air volume of central air conditioning is designed based on the fuzzy model. Through the experimental test, the energy-saving effect of the central air conditioning control system adopting traditional PID controller and the automatic control system based on the intelligent control algorithm is compared.
The results show that the application of fuzzy intelligent control algorithm improves the performance of the system, optimizes the energy efficiency of the central air conditioning system, and significantly reduces the power consumption of the system. However, in the performance test of this study, only the impact of personnel access times and temperature difference on power consumption of system is compared, and the data is relatively simple.

In the later stage, the test scope can be expanded to evaluate the system performance better. To sum up, the results of this study expand the application and development of intelligent control technology.

The application of intelligent control algorithm to variable air volume control system of central air conditioning has essential practical and positive significance, thus laying a foundation for the development of subsequent energy-saving buildings.

\section{References}

[1] Banihashemi S, Ding G, Wang J. Developing a hybrid model of prediction and classification algorithms for building energy consumption. Energy Procedia, 2017, 110, pp. 371-376.

[2] Amasyali K, El-Gohary N M. A review of datadriven building energy consumption prediction studies. Renewable and Sustainable Energy Reviews, 2018, 81, pp. 1192-1205.

[3] Shaikh P H, Nor N B M, Nallagownden P, et al. Intelligent multi-objective optimization for building energy and comfort management. Journal of King Saud University-Engineering Sciences, 2018, 30(2), pp. 195-204.

[4] Monaem Elmnifi, Moneer Alshilmany, Moftah Abdraba (2019). Potential of Municipal Solid Waste in Libya For Energy Utilization. Acta Mechanica Malaysia, 2(1): 11-15.

[5] S.K. Gugulothu, N. Prabhu Kishore, V. Phani Babu, Girish Sapre (2019). Computational Investigation of Mhd Free Convection on A Flat Vertical Plate Embedded with Micro Polar Fluid. Acta Mechanica Malaysia, 2(1): 16-22.

[6] Dayong Xu (2019). Research on Supply Chain Management Strategy of Longtang Electric Engineering Co. Ltd. Acta Electronica Malaysia, 3(1): 10-13.

[7] Shahajada Mahmudul Hasan, Shahed Hasan Khan Tushar, Md. Hafizur Rahman, Anisur Rahman Shimu (2019). An Optimized Design of Electromagnet and Float for A Magnetic Suspension System. Acta Electronica Malaysia, 3(1): 14-18.

[8] Nigar Ali, Saeed Ahmad, Sartaj Aziz, Gul Zaman (2019). The Adomian Decomposition Method For Solving Hiv Infection Model Of Latently Infected Cells. Matrix Science Mathematic, 3(1): 05-08. 
[9] Eltag K, Aslamx M S, Ullah R. Dynamic Stability Enhancement Using Fuzzy PID Control Technology for Power System. International Journal of Control, Automation and Systems, 2019, 17(1), pp. 234-242.

[10] Sun Y, Li S, Lin B, et al. Artificial neural network for control and grid integration of residential solar photovoltaic systems. IEEE transactions on sustainable energy, 2017, 8(4), pp. 14841495.

[11] Chua K H, Lim Y S, Morris S. A novel fuzzy control algorithm for reducing the peak demands using energy storage system. Energy, 2017,122 , pp. 265-273.

[12] Li J. Study on the Elman Neural Network Operation Control Strategy of the Central Air Conditioning Chilled Water System. World Journal of Engineering and Technology, 2019, 7(02), pp. 73.
[13] Sun L, Li Y, Zhang D, et al. Fuzzy radial basis function control for central air conditioning system. Electric Machines and Control, 2017, 21(5), pp. 110-116.

[14] Mohammed J A K, Mohammed F M, Jabbar M A S. Investigation of high performance split air conditioning system by using Hybrid PID controller. Applied Thermal Engineering, 2018, 129 , pp. 1240-1251.

[15] Kosaka T, Koyanagi K, Satake Y, et al. Simplification of Simulation Models for AirConditioning Controlling System. IEEJ Transactions on Electronics Information and Systems, 2017, 137(8), pp. 1001-1008.

[16] Liu M, Guo Q, Zhang G, et al. Application of emergency control of central air conditioning in safe and stable operation of large power grid. The Journal of Engineering, 2019, 2019(16), pp. 1539-1543. 